\title{
Erythrocyte glycophorins as receptors for Plasmodium merozoites
}

\author{
Ewa Jaskiewicz ${ }^{1,2^{*}}$, Marlena Jodłowska', Radosław Kaczmarek ${ }^{1}$ and Agata Zerka ${ }^{1}$
}

\begin{abstract}
Glycophorins are heavily glycosylated sialoglycoproteins of human and animal erythrocytes. In humans, there are four glycophorins: A, B, C and D. Glycophorins play an important role in the invasion of red blood cells (RBCs) by malaria parasites, which involves several ligands binding to RBC receptors. Four Plasmodium falciparum merozoite EBL ligands have been identified: erythrocyte-binding antigen-175 (EBA-175), erythrocyte-binding antigen-181 (EBA-181), erythrocyte-binding ligand-1 (EBL-1) and erythrocyte-binding antigen-140 (EBA-140). It is generally accepted that glycophorin A (GPA) is the receptor for P. falciparum EBA-175 ligand. It has been shown that a $(2,3)$ sialic acid residues of GPA O-glycans form conformation-dependent clusters on GPA polypeptide chain which facilitate binding. P. falciparum can also invade erythrocytes using glycophorin B (GPB), which is structurally similar to GPA. It has been shown that P. falciparum EBL-1 ligand binds to GPB. Interestingly, a hybrid GPB-GPA molecule called Dantu is associated with a reduced risk of severe malaria and ameliorates malaria-related morbidity. Glycophorin C (GPC) is a receptor for $P$. falciparum EBA-140 ligand. Likewise, successful binding of EBA-140 depends on sialic acid residues of N- and O-linked oligosaccharides of GPC, which form a cluster or a conformational structure depending on the presence of peptide fragment encompassing amino acids (aa) 36-63. Evaluation of the homologous P. reichenowi EBA-140 unexpectedly revealed that the chimpanzee homolog of human glycophorin D (GPD) is probably the receptor for this ligand. In this review, we concentrate on the role of glycophorins as erythrocyte receptors for Plasmodium parasites. The presented data support the long-lasting idea of high evolutionary pressure exerted by Plasmodium on the human glycophorins, which emerge as important receptors for these parasites.
\end{abstract}

Keywords: Erythrocyte glycophorins A, B, C, D, Plasmodium EBA merozoite ligands, Receptor-ligand interaction, Malaria resistance

\section{Background}

Glycophorins, major sialoglycoproteins of human and animal erythrocytes, are transmembrane type 1 glycoproteins with relatively low molecular weight $(20-30 \mathrm{kDa})$, carrying sialylated O-glycans and/or N-glycans $[1,2]$. Despite these similarities, glycophorins show interspecific as well as intraspecific structural differences [3-5]. Human erythrocytes carry four glycophorins identified so far: GPA, GPB, GPC and GPD (Table 1). The structure and genetics of human glycophorins, as well as their

\footnotetext{
*Correspondence: ewa.jaskiewicz@hirszfeld.pl

${ }^{1}$ Laboratory of Glikobiology, Ludwik Hirszfeld Institute of Immunology and Experimental Therapy, Polish Academy of Sciences, Rudolfa Weigla 12, 53-114 Wroclaw, Poland

Full list of author information is available at the end of the article
}

common and rare genetic variants, are well known and have been the subject of several reports [6-19].

Glycophorins carry blood group antigens, including MN, Ss and Gerbich [1, 2]. Most GPA and GPB O-glycans are NeuAc $\alpha 2-3$ Gal $\beta 1-3(N e u A c \alpha 2-6) G a I N A c-$ tetrasaccharide chains linked to serine or threonine residues $[15,16]$, but truncated O-glycans (e.g. monosialylated linear trisaccharide, sialylated or nonsubstituted GalNAc residues) are also present. It is generally assumed that all human glycophorins contain similar O-glycans, while GPA and GPC also contain N-glycosidic chains at Asn26 and Asn8 residues, respectively. The structures of both N-glycans have been determined [17-19] and they contain complex biantennary chains with a bisecting GlcNAc and terminal sialic acid residues. In addition, GPC $\mathrm{N}$-glycan contains small amounts of terminal fucose [19]. 
Table 1 Biochemical properties of human erythrocyte glycophorins [1, 2]

\begin{tabular}{|c|c|c|c|c|}
\hline \multirow[t]{2}{*}{ Glycophorin } & \multirow[t]{2}{*}{ No. of copies per cell } & \multirow[t]{2}{*}{ Length (aa)/MW (Da) } & \multicolumn{2}{|l|}{ Number of } \\
\hline & & & O-glycans & N-glycans \\
\hline A & $1 \times 10^{6}$ & $131 / 43,000$ & 16 & 1 \\
\hline B & $2 \times 10^{5}$ & $71 / 22,000$ & 11 & 0 \\
\hline C & $1.3 \times 10^{5}$ & $128 / 39,000$ & 12 & 1 \\
\hline $\mathrm{D}$ & $5 \times 10^{4}$ & $107 / 25,000$ & 6 & 0 \\
\hline
\end{tabular}

In recent years considerable progress has been made in the field of glycophorins as receptors for Plasmodium parasites. In particular, genome-wide association studies have shown that resistance to malaria may be connected with human glycophorin $A B E$ locus [20], suggesting that glycophorins play an important role in erythrocyte invasion by Plasmodium malaria parasites.

Invasion of erythrocytes by Plasmodium parasites is a multistep process involving several ligands which enable the merozoite to gain entry to red blood cells (RBCs) [21-24]. Proteins belonging to two families, erythrocyte binding-like (EBL) and reticulocyte binding-like (RBL), have been identified as major determinants of erythrocyte invasion $[25,26]$. Four functional EBL proteins have been found in P. falciparum merozoites, so far: erythrocyte-binding antigen-175 (EBA-175); erythrocyte-binding antigen-181 (EBA-181); erythrocyte-binding ligand-1 (EBL-1); and erythrocyte-binding antigen-140 (EBA-140) $[27,28]$. All EBA ligands are transmembrane proteins and consist of six regions (I-VI) in their ectodomains. Two of these regions, II and VI, contain several conserved cysteine residues. Region II (RII) of $P$. falciparum EBA proteins comprises two homologous DBL domains in tandem: F1 (aa 8-282) and F2 (aa 297-603); in contrast, RII of P.vivax erythrocyte binding proteins contain only one DBL domain.

Plasmodium falciparum EBA-175 ligand [29-32] was the first and is the best characterized protein of the EBL family, considered to be one of the most important $P$. falciparum merozoite invasion ligands $[32,33]$. It has been shown that EBA-175 is a target of human inhibitory antibodies present in sera of malarial patients, while animal antibodies recognizing EBA-175 can block merozoite invasion of RBCs [34-36]. Region II (616 aa) Pf EBA-175 was initially shown to mediate erythrocyte binding [31]. When truncated Regions I-VI of the Pf EBA-175 ectodomain were expressed on the surface of COS7 cells, only RII was bound by human erythrocytes in the rosetting assay. Furthermore, when domains F1 and F2 were expressed separately in COS cells, only F2 was shown to bind erythrocytes. The binding of F2 was similar to that of the entire Region II and to that of the full-length
EBA-175, proving that the F2 domain of RII alone can facilitate erythrocyte binding. Because RII is highly conserved among laboratory and field isolates, it is considered as a potential vaccine candidate [26].

Evaluation of high-priority antigens and their receptors may serve as a means to rational design of novel therapeutics, which can inhibit binding of merozoites to erythrocytes during the blood stage of malaria [26, 37, 38]. However, the redundancy of EBA and RBL ligands, which enable the merozoite to use alternative $\mathrm{RBC}$ receptors and thus alternative invasion pathways, is one of the major obstacles to block invasion in a strain-transcending manner [24-26]. In this review, we concentrate on the role of glycophorins as erythrocyte receptors for Plasmodium parasites in general, and EBL merozoite proteins in particular.

\section{Glycophorin A as the receptor for Plasmodium falciparum ligands}

It was shown that the EBA-175 ligand does not bind to erythrocytes treated with neuraminidase, which cleaves $\alpha(2,3)$-linked sialic acids from the glycophorin oligosaccharide chains. In addition, $P$. falciparum parasites are unable to invade erythrocytes that lack glycophorins $A$ and $B\left(M^{k}\right.$ phenotype). These data suggested that an erythrocyte membrane sialoglycoprotein (glycophorin A) may be the receptor for P. falciparum EBA-175 [29].

Moreover, binding of Pf EBA-175 to human GPBnegative erythrocytes (S-s-U-u-) and lack of binding to En(a-) erythrocytes, which are GPA-negative, confirmed that GPA is the receptor for EBA-175 ligand [31]. To investigate the binding site on GPA, tryptic (MT1 1-31, 1-39) and chymotryptic (MCH1 1-64, MCH2 1-34, $\mathrm{MCH} 3$ 35-64) glycopeptides of the extracellular domain of GPA were used in EBA-175 binding inhibition assay [31]. It was found that binding of recombinant EBA-175 was inhibited only by glycopeptide MCH1 (aa 1-64), which contains 16 O-linked sialotetrasaccharides. The tryptic peptides MT1, containing 11 of the 16 oligosaccharide chains, as well as chymotryptic peptides $\mathrm{MCH} 2$ and $\mathrm{MCH} 3$, did not reveal significant binding inhibition, suggesting that in addition to the sialic acid residues, the 
presence of polypeptide chain of GPA is necessary for binding. Moreover, it was shown that GPB, which shares the first 26 aa residues with GPA and has 11 identical oligosaccharide chains, did not block EBA-175 binding. These data provided evidence that aa 1-64 of GPA play an important role in interactions with the ligand. Moreover, treatment of erythrocytes with $\mathrm{N}$-glycanase did not affect rosetting of erythrocytes, demonstrating that the $\mathrm{N}$-linked oligosaccharide does not play a role in EBA-175 binding. In summary, it is now generally assumed that GPA is the receptor for $P$. falciparum EBA-175 ligand, and $\alpha(2,3)$ sialic acid residues of O-linked glycosaccharides form conformation-dependent clusters on the GPA polypeptide chain which facilitate binding. However, other studies that included flow cytometry and ELISA analyses provided evidence that the EBA-175 ligand may also bind to desialylated GPA by engaging a 21-aa fragment (aa 1076-1096) of Region III and IV [39]. Evaluation of 11 truncated peptides of EBA-175 revealed that the peptide comprising aa 1085-1096 contains the erythrocyte binding site and strongly inhibits parasite invasion. Thus, it may be hypothesised that this EBA-175 peptide takes part in the second step of erythrocyte binding, which is a two-step phenomenon. The first step of this process is the initial binding of RII fragment to sialic acids of GPA, which induces a conformational change in EBA-175, which in turn exposes peptide (aa 1076-96) to subsequent erythrocyte binding. The ability of EBA-175 antigen to bind unsialylated GPA was later confirmed by other studies [40, 41].

The crystal structure of the erythrocyte binding domain of Pf EBA-175 (Region II) was resolved at $2.3 \AA$ resolution, giving insight into the molecular mechanisms underlying the GPA binding [42]. The RII domain of Pf EBA-175 was crystallised in two distinct crystal forms, either as a monomer or as a homodimer, where two RII molecules arranged in an antiparallel fashion interact with each other in a manner resembling a handshake.

The centre of the homodimer contains two channels; most of the residues forming the channel surface come from the two F2 domains of the dimer, confirming its dominant role in the binding site of the EBA-175 ligand. In order to propose a model for the binding, the authors co-crystallized Region II with a glycan ( $\alpha-2,3-$ sialyllactose) that may be considered as an analogue of Neu5Ac( $\alpha 2,3)$-Gal (the glycan receptor required for binding). The dimer interface contains six glycan binding sites: four of them are located inside the channel, while the remaining two are exposed in a cavity on the top surface. All of the six glycans contact residues of both monomers of Region II, implicating that binding of the receptor depends on dimerization. Computer modelling suggested that the $\mathrm{O}$-glycosidic sialotetrasaccharides of
GPA would fit in the binding sites with good geometry. A mutational analysis of RII suggested that all of the six glycan binding sites are necessary for RII binding. All these data helped to create a binding model of EBA-175 to GPA in which the binding induces dimerization of RII by assembling around the dimeric GPA extracellular domain. Alternatively, the GPA monomers could be bound to the outer surface of the RII dimer providing glycans for binding cavities.

The molecular basis of GPA and EBA-175 interaction involving the glycan moiety $[\operatorname{Neu} 5 \mathrm{Ac}(\alpha 2,3)-\mathrm{Gal}]$ is of particular interest when novel malarial therapeutics are considered. Indeed, it was shown that the sugar moiety, 2,3-didehydro-2-deoxy- $N$-acetylneuraminic acid (DANA), a structural analog of $N$-acetylneuraminyl$\mathrm{N}$-acetate-lactosamine, is a good inhibitor of Pf EBA175 - GPA interaction ( $88 \%$ of inhibition at $100 \mu \mathrm{M})$ [43]. Moderate inhibition was also observed for monomers or oligomers of $\mathrm{N}$-acetylneuraminic acid (from 25 to $68 \%$ at $100 \mu \mathrm{M})$. Moreover, it was shown that DANA is able to significantly inhibit erythrocyte invasion by $P$. falciparum. Thus, such compounds may one day be used as part of drug cocktails to reduce disease severity [43, 44].

A successful attempt to prevent formation of EBA175-GPA complex involved the employment of monoclonal antibodies recognizing epitopes at glycan binding sites of RII/EBA-175 [45, 46]. Quantitative binding analysis revealed that a recombinant ectodomain of EBA-175 obtained in HEK cells interacts with GPA in a Neu5Ac(2-3)Gal-dependent manner with $K_{D} 0.26$ $\mu \mathrm{m}$ whereas a recombinant RII fragment alone bound GPA with a lower affinity [47]. Moreover, binding of RII to GPA and Neu5Ac(2-3)Gal showed similar affinity, whereas the full-length ectodomain of EBA-175 bound GPA with 2-fold higher affinity than the glycan alone. These results support the hypothesis that the RII fragment interacts with the GPA glycan moieties, but other extracellular regions of EBA-175 ligand outside of RII domain also engage the polypeptide of GPA.

Recombinant GPA, produced in a mammalian expression system [48], was used to identify the GPA-RII binding region and evaluate the importance of its glycans for this interaction (Fig. 1). A recombinant GPA lacking the polypeptide fragment encoded by exon 3 did not bind EBA-175/RII. It was shown that presence of GPA multiple glycans is a prerequisite for high-avidity binding. Moreover, the glycosylation mutants had different effects on the binding. The triple glycan mutant, missing three glycans otherwise attached to Ser66, Ser69 and Thr72, did not bind to EBA-175 RII at all. On the other hand, the GPA individual mutants S69A and T72A revealed significantly reduced binding in comparison to S66A mutant, which bound with only minimally lower affinity than the 


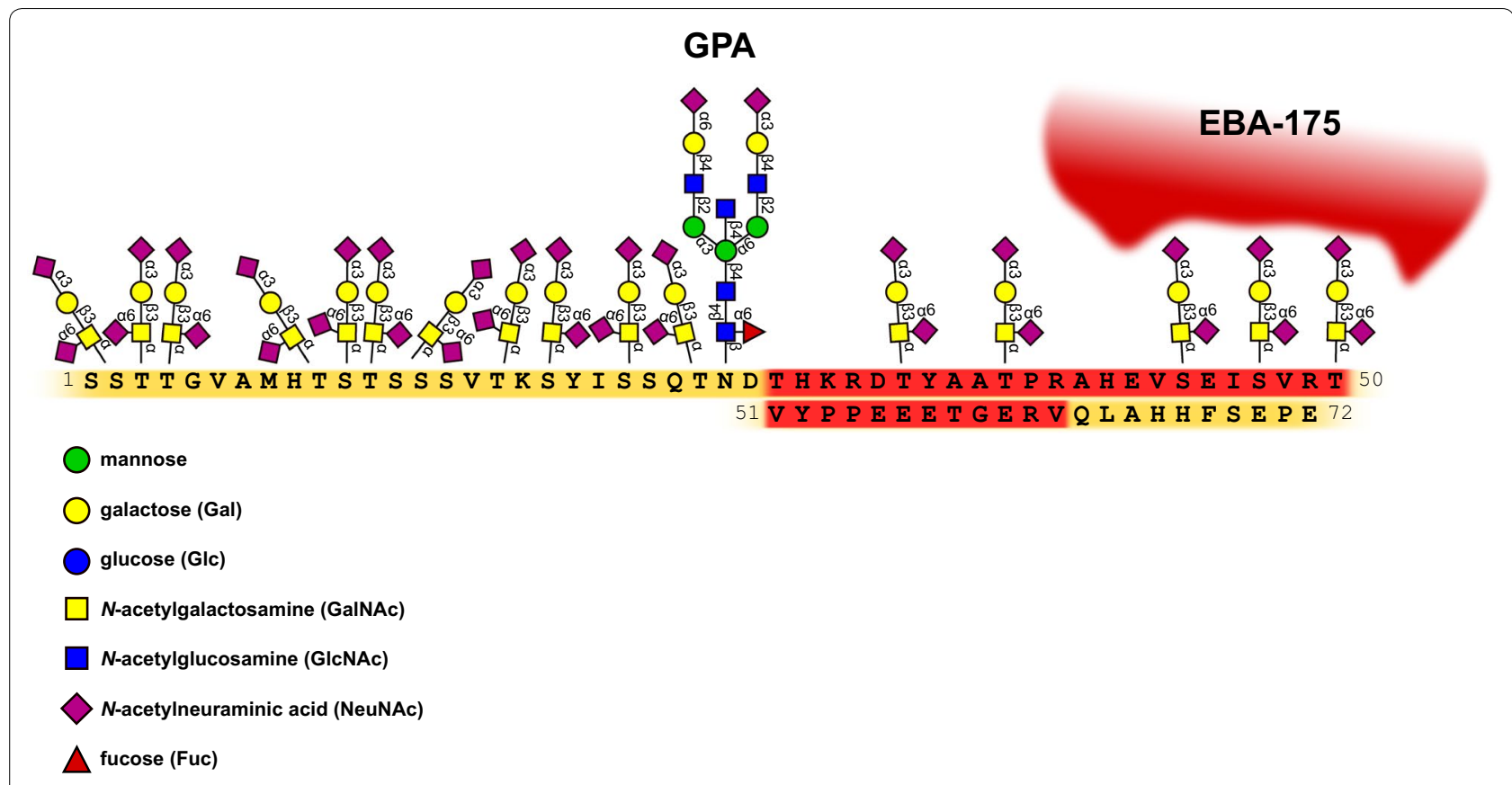

Fig. 1 Amino acid sequence of external (aa 1-72) domain of GPA, its glycan structures and attachment sites [1, 4 modified]. GPA polypeptide fragment encoded by exon 3 is marked in red and its O-glycans recognized by EBA-175 ligand are shown

wild-type GPA. Together, these results suggest that the three glycans of GPA polypeptide fragment encoded by exon 3 are critical for high-avidity binding of EBA-175 to GPA on erythrocytes. They are also consistent with the findings discussed earlier [31] demonstrating that digestion of purified, native GPA by trypsin (Arg39) within the sequence encoded by exon 3 abolishes its ability to inhibit erythrocyte rosette formation. In addition, it was shown before that $\mathrm{M}^{\mathrm{g}}$ erythrocytes, in which GPA lacks glycans at aa residues 24, 25 and 26 [49], can be invaded by $P$. falciparum [50]. However, in other studies, a high frequency of variants with polymorphisms within exon 2 of the GPA gene was identified in three continental groups: sub-Saharan Africans, South Asians and Europeans. These results suggest that balancing selection has influenced exon 2, but not exon 3 of the GPA gene, in $P$. falciparum endemic regions [51].

Recently, new data emerged demonstrating an essential role of GPA-band 3 complex during the initial adhesion phase of malaria parasite invasion on RBCs [52]. It was shown that MSP1, which is highly abundant on the merozoite surface, binds to the trypsin-resistant (aa 31-72) extracellular domain of GPA. Moreover, deficiency of the Band 3-GPA-protein 4.2 complex in mouse RBCs was shown to be sufficient factor for resistance to invasion by $P$. yoelli. A similar effect was obtained for Band 3-GPA-band 4.2 null mouse RBCs infected with $P$. falciparum 3D7 strain in vitro. Based on these results, it was hypothesized that merozoites may use ligands that bind Band 3 (MSP1) and GPA (EBA 175) simultaneously, thus integrating distinct invasion pathways. Additionally, these findings suggested that MSP binding to GPA may change the RBCs membrane properties during parasite invasion [53].

These changes in the deformability of RBCs caused by EBA-175 binding to GPA were shown to activate phosphorylation of the cytoskeleton, which alters properties of the erythrocyte membrane and is required for successful invasion [54].

The most recent data concerning EBA-175-GPA interaction revealed its new role in promoting clustering of RBCs by EBA-175 antigen, shed post invasion, which is dependent on GPA [55]. This clustering not only provides merozoites ready access to uninfected RBCs but also protects from immune recognition. This phenomenon leads to enhanced merozoite growth and their immune evasion.

In summary, the major invasion and evasion pathway of P. falciparum merozoites involves the interaction of EBA175 and GPA. The observed patterns of variation in the GPA gene suggest that GPA has been subject to balancing selection in populations living in the malaria endemic areas of Africa and in Europeans [56]. However, when GPA is not accessible, $P$. falciparum can invade erythrocytes using different pathways, which may involve other glycophorins present on the surface of erythrocytes. One 
of them is GPB (Fig. 2), which is similar to GPA and has identical first 26 aa residues and O-glycosidic chains [1, 2].

\section{Glycophorin B as the receptor for Plasmodium falciparum EBL-1 ligand}

Some isolates that depend on GPA for invasion can switch their invasion pathway in culture and invade GPAdeficient cells [57]. It was shown that P. falciparum can use an alternate pathway of invasion to enter human RBCs treated with trypsin, which digests GPA and GPC but not glycophorin B (GPB) (Fig. 2) [58]. Indeed, strains such as 3D7, HB3, Dd2 showed greatly reduced invasion rates for trypsin-digested S-s-U-RBCs (that lack GPB), indicating that the invasion depends on GPB [59]. However, the parasite ligand that binds to GPB remained unknown [60].

The early experiments showed that peptide fragments of EBL-1 merozoite ligand bound on human RBC membrane to a protein with an apparent molecular weight of $36 \mathrm{kDa}$ [61]. It was also suggested that EBL-1 ligand can bind to GPB because its recombinant RII expressed on CHO-K1 cells did not bind to S-s-U RBCs, which lack GPB. In addition, the EBL-1 ligand immunoprecipitated from $P$. falciparum culture supernatant did not bind to S-s-U- RBCs. Moreover, EBL-1 ligand failed to bind to RBCs treated with chymotrypsin, which cleave GPB and neuraminidase, which removes sialic acid residues [62]. Thus, GPB emerged as a major candidate for EBL-1 binding. Statistical association between the presence of the $S$ allele, encoding $S$ antigen defined by presence of Met 29 residue in $\mathrm{GPB}[1,10]$ and the infection rate suggested that GPB is a key receptor in the Brazilian Amazon regions [63]. This finding was supported by the unusually high occurrence of GPB-null phenotype in the Efe Pygmies in the Democratic Republic of Congo [64].
Similar to EBA-175 the RII region of EBL-1 ligand contains two DBL domains, F1 and F2. The major binding site of EBL-1 for GPB was identified within its 69-amino acid segment (F2i) of its F2 domain [65]. Both F1 and F2 domain and the F2i fragment of EBL-1 ligand partially inhibited the merozoite invasion of human RBCs. This result was confirmed by the finding that several peptides located within F1 and F2 domains of EBL-ligand bind to human RBCs [61]. Another proof that GPB is an important receptor for $P$. falciparum invasion came from screening of the merozoite and human RBC proteomes [66]. Of 13 predicted peptides, one peptide (SYTIRRLIKA) derived from human GPB showed the ability to inhibit parasite growth. These data may suggest that synthetic peptides may be considered to be included in the multi-subunit malaria vaccine [65].

Recently, using ex vivo cultured erythrocytes (cRBCs) with decreased expression of GPA, GPB or GPC, it was shown that most laboratory strains and field isolates of P. falciparum require GPB for invasion [67]. In the case of field isolates, GPB was found to be similarly important for invasion as GPA. Importantly, the observation that GPB is a key receptor in P. falciparum invasion is in contradiction to the fact that some laboratory strains have mutated or deleted the ebl-1 gene [67].

\section{Glycophorin A-B hybrid (Dantu) as the receptor for $P$. falciparum}

GPB-null phenotype is prevalent in the regions where malaria is endemic [68], suggesting that evolutional pressure exerted by $P$. falciparum caused selection for this polymorphism. Thus, the Dantu variant of GPB, which is a hybrid GPB-GPA molecule with a GPB N-terminus and GPA C-terminal region, seems to be responsible for protection against $P$. falciparum invasion in vitro $[68,69]$.

The large genetic association study (GWAS) of malaria, which included over 11,000 African children, revealed

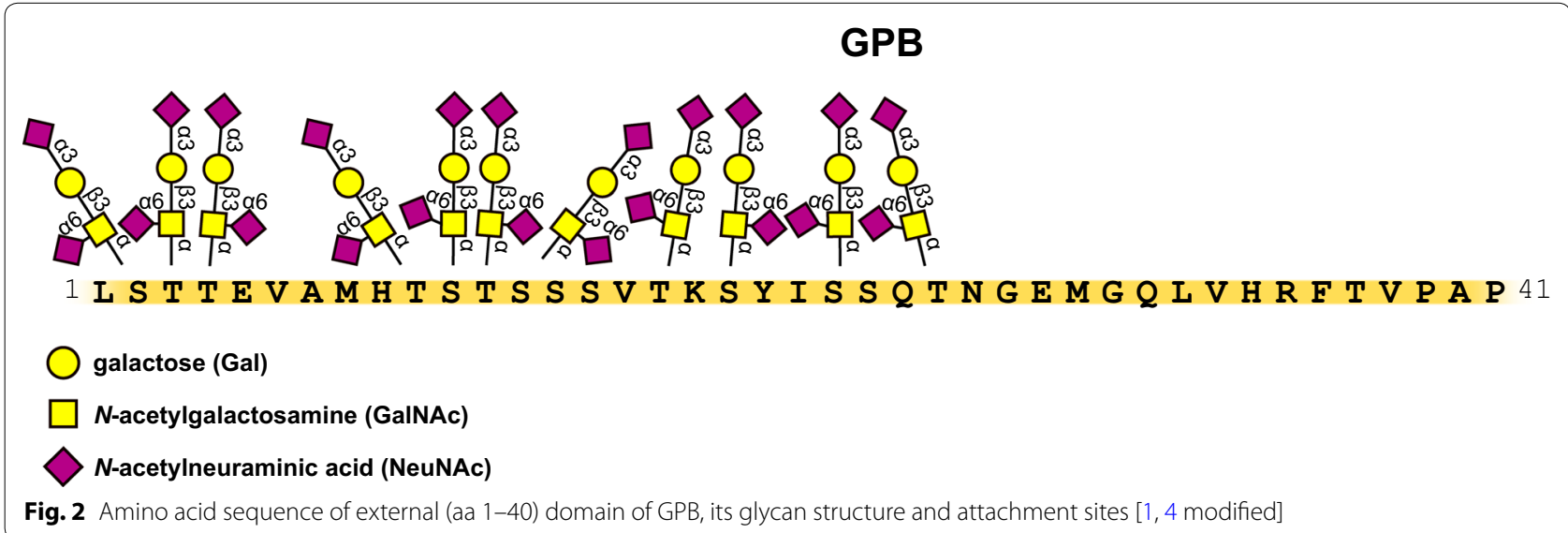


a new locus of resistance to severe malaria [70]. It was found close to the cluster of glycophorin genes GPE, $G P A$ and $G P B$. Moreover, evaluation of genome sequence data from individuals of sub-Saharan descent suggested a strong protective effect of the Dantu (NE type) genotype against cerebral malaria anemia in East Africa [71]. The Dantu NE chromosome model contains GypE, GypB and GypA glycophorin genes; if translated, the encoded protein joins the extracellular domain of GPB to the transmembrane and intracellular domains of GPA, since GPE is probably not expressed at the protein level [72]. It creates the peptide sequence of Dantu NE blood group antigen, called DUP4. In addition to the Dantu variant, multiple deletions at the GPE-GPB-GPA locus were identified. The absence of exon 3 in cDNA of GPB and GPE limits specific recognition of GPA to EBA-175 merozoite ligand. One may even speculate that GPB and GPE arose due to selective pressure because the binding site on GPA has been lost.

The most recent studies of gene polymorphisms in children in Kenya confirmed that the presence of Dantu mutation was associated with reduced risk of severe malaria by $43 \%$ among heterozygotes and $74 \%$ among homozygotes, and the protection was equal for all forms of severe malaria. In addition, Dantu seemed to ameliorate malaria-related morbidity [73]. Moreover, studies in Tanzania indicated that DUP4 is susceptible to somatic variation and may be associated with increased haemoglobin levels [74]. These findings raised the question of how DUP4 protects against malaria. It may be hypothesized that it could affect interactions between $P$. falciparum receptors and host Band 3 at the RBC surface [73].

\section{Glycophorin C as the receptor for Plasmodium ligands}

Studies on the EBA-140 ligand, which similarly to EBA175 contains two DBL domains F1 and F2 [27], showed that this ligand does not bind neuraminidase-treated human RBCs [75, 76]. However, RBCs lacking GPA [En(a)], GPB (S-s-U-), or GPA and GPB [Dombrock, Gy(a-b-)] proved to bind EBA-140 effectively. Other RBC variants of the Yus and Gerbich phenotype, encoded by the genes lacking exons 2 or 3 in the GYPC gene [4] resulting in deletion of GPC amino acid residues 17-35 and 36-63, respectively, showed markedly reduced binding [75]. Moreover, only RBCs of the Leach phenotype (GPC null), lacking GPC, were not bound by EBA-140 ligand. Thus, these data suggested that the EBA-140 ligand binds human RBCs through GPC (Fig. 3). However, some contradictory results showed that (depending on the parasite

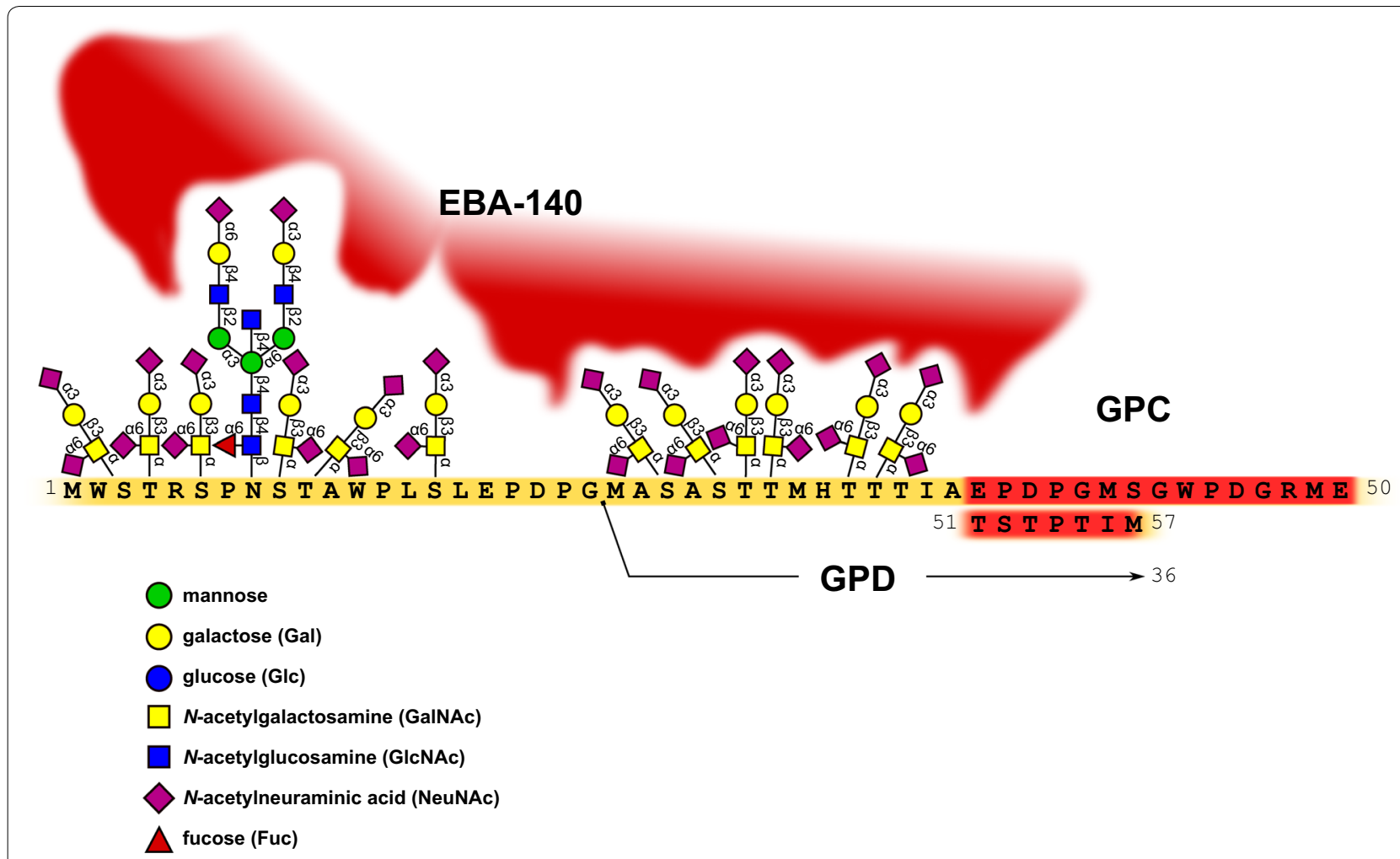

Fig. 3 Amino acid sequence of external (aa 1-57) domain of GPC and GPD (aa 1-36) with their glycan structure and attachment sites [1, 4 modified]. GPC/GPD polypeptide fragment encoded by exon 3 is marked in red and putative glycans recognized by EBA-140 ligand are shown 
strain) EBA-140 binding was not affected or only partially abolished when erythrocytes were treated with trypsin, which removes extracellular parts of glycophorins A and C $[76,77]$.

These inconsistencies may be attributed to the polymorphisms within the binding region of $P$. falciparum EBA-140 in different strains, which have been reported to alter host receptor specificity [78]. Five polymorphisms in the EBA-140 RII of different strains of $P$. falciparum have been identified (V,S,T,K; V,S,K,K; I,S,K,K; $\mathrm{I}, \mathrm{N}, \mathrm{R}, \mathrm{E}$; and $\mathrm{I}, \mathrm{N}, \mathrm{K}, \mathrm{K})$, which encompass four amino acid residues of the R II F1 domain in positions 185, 239, 261 and 285, respectively. These polymorphic variants of EBA-140 R II expressed on the surface of COS cells showed different receptor binding specificity. Of these five variants, only RII from the $\mathrm{Dd} 2 / \mathrm{Nm}$ strain $(\mathrm{V}, \mathrm{S}, \mathrm{T}, \mathrm{K})$ was sensitive to neuraminidase and trypsin treatment of RBCs, and was the only one which did not bind to Gerbich-negative RBCs. A different binding pattern of two RII/EBA-140 polymorphic variants V,S,T,K and V,S,K,K was also confirmed in another study when both RII variants were expressed on the surface of Chinese hamster ovary (CHO-K1) cells [79]. It was demonstrated that only $\mathrm{V}, \mathrm{S}, \mathrm{T}, \mathrm{K}$ (but not $\mathrm{V}, \mathrm{S}, \mathrm{K}, \mathrm{K}$ ) variant does not bind to neuraminidase treated RBCs or to Gerbich-negative erythrocytes. These data suggest that only one amino acid, T261 in V,S,T,K variant, may be the key residue for sialic aciddependent GPC binding.

On the other hand, a study using native EBA-140 ligands from $P$. falciparum culture supernatants provided contradictory evidence [80]. All of the five polymorphic EBA-140 variants were proven to bind to GPC in a Western blotting assay with human erythrocyte membranes. None of these ligands bound to neuraminidase-treated or trypsin-treated RBCs or to Gerbich, Yus, Leach or Yus/ Gerbich erythrocytes, which contain GPC variant forms or lack GPC completely. The authors came to the conclusion that the polymorphisms in EBA-140/R II does not change its specificity, but rather alter the affinity of binding to its receptor, which is GPC, and subsequently determine their ability to invade using this receptor. The discordant results obtained in the binding studies described above may be attributed to the use of different methodology. In contrast to the previous studies, which were based on recombinant forms of EBA-140 R II expressed on COS7 [78] or CHO-K1 [79], this study utilised native EBA-140 ligand from culture supernatants of different $P$. falciparum strains [80].

A decreased binding of EBA-140 observed in the case of Gerbich-negative erythrocytes [80-82] implied that the peptide encoded by exon 3 of GPC gene (aa 36-63) is of particular significance. However, these results were not confirmed in another study using EBA-140 from 3D7 clone supernatant $(\mathrm{I}, \mathrm{N}, \mathrm{K}, \mathrm{K})$, since this variant bound to Gerbich erythrocytes with a moderately higher efficiency than normal RBCs [83]. At the same time, the binding of EBA-140 to Yus cells was found to be decreased. It suggested that the amino acid residues 17-35, which Yus erythrocytes lack, but not 36-63, as previously suggested, play a significant role in invasion. However, what seemingly supports the hypothesis that aa 36-63 of GPC are of significance in merozoite invasion is the fact that frequency of the allele underlying Gerbich negativity has increased to almost $50 \%$ in populations where malaria is endemic, such as New Guinea [84]. Since P. falciparum parasites invade Ge-negative cells less efficiently than normal erythrocytes, it is highly possible that selection for this allele has been an evolutionary mechanism of protection against malaria. Determining which fragment of the polypeptide chain of GPC is responsible for binding EBA-140 ligand was the subject of several studies, also in our laboratory where we used RII/EBA-140 expressed in a baculovirus system. It confirmed that the GPC region encompassing aa residues $36-63$ is indispensable for EBA-140 ligand binding [82].

Another issue to be solved is the role of the oligosaccharide chains of GPC in EBA-140 binding. The extracellular domain of GPC is believed to have 12 sialylated $\mathrm{O}$-glycosidic chains linked to serine and threonine residues and one complex $\mathrm{N}$-glycosidic chain at Asn8 $[1,2]$. It was found that the N-linked glycan is crucial for EBA140 binding of GPC [85]. It has been proposed that sialic acid residues of closely spaced $\mathrm{N}$-linked and O-linked oligosaccharides of GPC form a cluster or a conformational structure that provides multivalency for the successful binding of EBA-140 (Fig. 3).

The turning point in the search for better understanding of the molecular basis of invasion by EBA-140 ligand came with solving the crystal structure of its recombinant binding region [86, 87]. It was shown that RII/EBA-140 is present as a monomer in solution in the asymmetric unit. The crystal structure of RII/EBA-140 bound to sialyllactose confirmed that this ligand-receptor complex exists as a monomer in solution, consistent with the crystal structures of unbound RII. Moreover, modelling revealed two receptor glycan-binding sites, each of them located in the F1 and F2 domain, in a structurally similar position. The two binding sites are present in a region that contains a high concentration of positively charged residues, presumably allowing the interaction with sialic acid which enters inside the binding pocket. It was suggested that domains F1 and F2 of R II/ EBA-140 act distinctly, and their role in receptor binding is different. It seems reasonable to assume that although both domains may be considered as required for binding, only domain F1 is involved in a direct interaction with the receptor. 
In comparison to other RII regions of the DBL family, including homologous $P$. falciparum EBA-175 and $P$. vivax DBL ligand, the structure of PfEBA-140 RII and its glycan binding mechanism of PfEBA-140 is unique [87]. It was shown that DBL-domain receptor-engagement and glycan binding by PvDBP and PfEBA-175 ligands requires their dimerization $[42,88]$. In addition, the PvDBP receptor binding site is formed through dimerization of the ligand upon receptor (DARC) binding $[88,89]$. The formation of a homodimer is critical for and driven by receptor engagement. Consistently, RII-PfEBA-175 is known to exist as a monomer and dimerization is induced when binding with its receptor, GPA, occurs. Unlike the latter, RII of the PfEBA-140 ligand exists only in a monomeric form and consequently can bind only two GPC glycan moieties, whereas the PfEBA-175 RII dimer interacts with six O-linked glycans of GPA. These characteristics likely contribute to the increased affinity of the ligands to their receptors, GPC and GPA, respectively. Thus, whether Plasmodium ligands engage receptors as monomers or dimers is an issue of profound implications for parasite biology and control [42].

The structures of GPC N-glycans essential for GPCEBA-140 binding were elucidated using mass spectrometry [90]. It was shown that apart from the complex-type sialylated structures, many GPC N-glycans contain terminal $\mathrm{H} 2$ blood antigen as well as polylactosamine chains with terminal fucose. It was hypothesized that presence of terminally fucosylated lactosamine units present in Gerbich-type GPC may explain the lack of EBA-140 binding to GPC Gerbich variant. Thus, it supported the theory about the crucial role of GPC sialylated N-glycan for GPC-EBA-140 interaction [85]. Moreover, it was shown that the $P$. falciparum EBA-140 ligand bound neither to Gerbich GPC deletion variant (lacking aa 36-63) nor to GPD (a truncated form of GPC lacking aa 1-21), but did bind to GPC Yus variant (lacking aa 17-35) [82]. It suggests that the heavily $\mathrm{O}$-glycosylated $\mathrm{N}$-terminal fragment of GPC (which also contains N-glycan) (Fig. 3) seems to be the binding site for EBA-140. It may be argued that deletion of the region including aa 36-63 in GPC Gerbich type, which in fact lacks O-glycans, might change the structure of GPC and influence the availability of other O-glycans and N-glycan attached to GPC. The essential role of sialylated oligosaccharide chains on GPC in EBA-140 binding is generally established, but the participation of individual O-glycans was not examined. In addition, the lack of EBA-140 binding to similarly glycosylated GPA (one N-glycan and 12-16 O-glycans) firmly suggests that GPC polypeptide also participates in the binding, either directly or by ensuring an aviability of glycans [82]. The crucial role of GPC N-terminus has been further corroborated by the prevalence of the rare Gerbich phenotype (Ge:-2,-3,4) in Papua New Guinea, which a $P$. falciparum endemic region [81, 84]. The molecular basis of these interactions may one day be a valuable means in the design of $P$. falciparum binding inhibitors for therapy.

In addition to the studies with the human P. falciparum parasite, interesting data recently came from studies using $P$. berghei. Because there is a significant homology in GPC genes across the species, the mouse GPC homolog was identified as a $\mathrm{RBC}$ receptor [91]. It was shown that the absence of GPC on mouse stem cellderived RBCs resulted in a significant decrease of invasion rate by the mouse parasite (up to 66\%) [91]. One homologous sequence to $P$. falciparum EBA-140 ligand in the $P$. berghei genome was identified. The putative protein called PBANKA-1332700 may serve as ligand for mouse GPC.

Once the parasite invades RBCs it releases variant surface antigens (var), such as erythrocyte membrane protein 1 (EMP1), STEVOR and RIFIN [92, 93]. Varencoded proteins travel to the RBC plasma membrane and bind host proteins on uninfected RBCs and endothelium, which leads to sequestration of infected RBCs [93]. In addition, the rosettes form aggregates of uninfected and infected RBCs, which protect infected RBCs from the environment [94]. However, the importance of the rosetting occurrence in vivo remains unrecognized. It was revealed that GPC mediates rosetting by $P$. falciparum STEVOR protein [95]. Indeed, soluble GPC inhibits binding of STEVOR to RBCs simultaneously preventing rosetting. Moreover, GPC deletion significantly reduces the ability of RBCs to form rosettes.

Although most RBCs rosetting experiments were performed with $P$. falciparum infected RBCs, the general rule applies to $P$. vivax as well and it may also involve GPC. Surprisingly, rosettes formed by $P$. vivax occur preferably among normocytes (mature RBCs) and not reticulocytes [96], although $P$. vivax normally prefers to invade reticulocytes. In addition, a strong inhibition of $P$. vivax rosette formation by an antibody against the $\mathrm{N}$-terminus of GPC was shown. Thus, GPC seems to function in the rosetting of $P$. vivax.

Glycophorin $D$ as the receptor on chimpanzee erythrocytes As discussed above, there is a general agreement that GPC but not GPD is $P$. falciparum EBA-140 receptor on human RBCs [79, 81-83, 97, 98]. However, evaluation of the binding of the homologous $P$. reichenowi EBA-140 ligand binding to chimpanzee RBCs [99] unexpectedly demonstrated that the chimpanzee homolog of human GPD is probably the receptor for that ligand $[4,100]$. It may be hypothesized that GPD is an ancestral EBA-140 receptor in non-human primates, while GPC emerged as 
a new receptor for $P$. falciparum in humans. This hypothesis needs to be corroborated using EBA-140 ligands from other species of Plasmodium invading Great Apes.

\section{Conclusions}

Given the high death toll caused by malaria since time immemorial, it is not surprising that human genetic variants that protect against malaria are common in populations living in tropical areas. One example is the sickle cell allele that protects from malaria in heterozygous carriers, or Duffy antigen, which is a receptor for P. vivax that has been lost in many African ethnic groups rendering them resistant to $P$. vivax infection. Plasmodium falciparum, which causes most malaria cases in Africa, targets heavily glycosylated proteins that cover the surface of human RBCs, named glycophorins. Although GPA, GPB and GPC have been known as P. falciparum receptors for quite some time, data linking their polymorphism with resistance to malaria have been missing. With the identification of specific protective alleles in the glycophorin locus of individuals resistant to severe malaria, glycophorins have moved to the forefront of research on malaria epidemiology. Moreover, these data finally consolidate the long-standing idea that the human glycophorin makeup is a footprint of high evolutionary pressure exerted by Plasmodium parasites. Altogether, glycophorins emerge as not only an important foothold for the parasite on its way into the RBC but also an essential step in our quest to fully understand the Plasmodium biology and design new malaria interventions.

\section{Abbreviations}

GPA: glycophorin A; GPB: glycophorin B; GPC: glycophorin C; GPD: glycophorin D; aa: amino acids.

\section{Acknowledgements}

We are indebted to Marcin Czerwinski for his detailed and critical reading of the manuscript.

\section{Authors' contributions}

EJ and MJ wrote the manuscript. RK proofed the manuscript and prepared all figures. AZ proofed the manuscript. All authors read and approved the final manuscript.

\section{Funding}

This publication was supported by Grants: Preludium UMO-2016/23/N/ NZ6/01462 and Harmonia UMO-2018/30/M/NZ6/00653 from the National Centre of Science, Poland.

\section{Availability data and materials}

All data presented and analyzed during this study are included in this published article.

\section{Ethics approval and consent to participate}

Not applicable.

\section{Consent for publication}

Not applicable.

\section{Competing interests}

The authors declare that they have no competing interests.

\section{Author details}

${ }^{1}$ Laboratory of Glikobiology, Ludwik Hirszfeld Institute of Immunology and Experimental Therapy, Polish Academy of Sciences, Rudolfa Weigla 12,

53-114 Wroclaw, Poland. ${ }^{2}$ Faculty of Biological Sciences, University of Zielona Góra, Szafrana 1, 65-516 Zielona Góra, Poland.

Received: 19 April 2019 Accepted: 19 June 2019

Published online: 24 June 2019

\section{References}

1. Lisowska E. Antigenic properties of human erythrocyte glycophorins. Adv Exp Med Biol. 1988;228:265-315.

2. Chasis JA, Mohandas N. Red blood cell glycophorins. Blood. 1992;80:1869-79.

3. Rearden A, Phan H, Kudo S, Fukuda M. Evolution of the glycophorin gene family in the hominoid primates. Biochem Genet. 1990;28:209-22.

4. Jaskiewicz E, Peyrard T, Kaczmarek R, Zerka A, Jodlowska M, Czerwinski $M$. The Gerbich blood group system: old knowledge, new importance. Transfus Med Rev. 2018:32:111-6.

5. Huang $\mathrm{CH}$, Xie SS, Socha W, Blumenfeld OO. Sequence diversification and exon inactivation in the glycophorin A gene family from chimpanzee to human. J Mol Evol. 1995;41:478-86.

6. Siebert PD, Fukuda M. Human glycophorin A and B are encoded by separate, single copy genes coordinately regulated by a tumor-promoting phorbol ester. J Biol Chem. 1986;261:12433-6.

7. Siebert PD, Fukuda M. Molecular cloning of human glycophorin B cDNA: nucleotide sequence and genomic relationship to glycophorin A. Proc Natl Acad Sci USA. 1987:84:6735-9.

8. Fukuda M. Molecular genetics of the glycophorin A gene cluster. Semin Hematol. 1993;30:138-51.

9. Rearden A, Magnet A, Kudo S, Fukuda M. Glycophorin B and glycophorin E genes arose from the glycophorin A ancestral gene via two duplications during primate evolution. J Biol Chem. 1993;268:2260-7.

10. Blumenfeld $\mathrm{OO}$, Huang $\mathrm{CH}$. Molecular genetics of the glycophorin gene family, the antigens for MNSs blood groups: multiple gene rearrangements and modulation of splice site usage result in extensive diversification. Hum Mutat. 1995;6:199-209.

11. Cartron JP, Le Van Kim C, Colin Y. Reid ME, Spring FA. Molecular basis of glycophorin C variants and their associated blood group antigens. Transfus Med. 1994;4:139-46.

12. Jaskiewicz E, Czerwinski M, Colin Y, Lisowska E. Recombinant forms of Gerbich blood group antigens: expression and purification. Transfus Clin Biol. 2002;9:121-9.

13. Blanchard D, Dahr W, Hummel M, Latron F, Beyreuther K, Cartron JP. Glycophorins $B$ and $C$ from human erythrocyte membranes. Purification and sequence analysis. J Biol Chem. 1987;262:5808-11.

14. Tomita M, Marchesi VT. Amino-acid sequence and oligosaccharide attachment sites of human erythrocyte glycophorin. Proc Natl Acad Sci USA. 1975;72:2964-8.

15. Fukuda M, Lauffenburger M, Sasaki H, Rogers ME, Dell A. Structures of novel sialylated O-linked oligosaccharides isolated from human erythrocyte glycophorins. J Biol Chem. 1987;262:1952-7.

16. Pisano A, Redmond JW, Williams KL, Gooley AA. Glycosylation sites identified by solid-phase Edman degradation: O-linked glycosylation motifs on human glycophorin A. Glycobiology. 1993;3:429-35.

17. Yoshima H, Furthmayr H, Kobata A. Structures of the asparagine-linked sugar chains of glycophorin A. J Biol Chem. 1980;255:9713-8.

18. Jaskiewicz E, Lisowska E, Lundblad A. The role of carbohydrate in blood group N-related epitopes recognized by three new monoclonal antibodies. Glycoconiugate J. 1990;7:255-68.

19. Ashline DJ, Duk M, Lukasiewicz J, Reinhold VN, Lisowska E, Jaskiewicz E. The structures of glycophorin C N-glycans, a putative component of the GPC receptor site for Plasmodium falciparum EBA-140 ligand. Glycobiology. 2015;25:570-81.

20. Winzeler EA. Glycophorin alleles link to malaria protection. Science. 2017;356:1122-3. 
21. Gaur D, Mayer DCG, Miller LH. Parasite ligand-host receptor interactions during invasion of erytrocytes by Plasmodium falciparum. Int J Parasitol. 2004;34:1413-29.

22. Cowman AF, Crabb BS. Invasion of red blood cells by malaria parasites. Cell. 2006;24:755-66.

23. Gaur D, Chitnis CE. Molecular interactions and signaling mechanisms during erythrocyte invasion by malaria parasites. Curr Opin Microbiol. 2011;14:22-8.

24. Cowman AF, Tonkin CJ, Tham WH, Duraisingh MT. The molecular basis of erythrocyte invasion by malaria parasites. Cell Host Microbe. 2017;22:232-45.

25. Tham W-H, Healer J, Cowman AF. Erythrocyte and reticulocyte bindinglike proteins of Plasmodium falciparum. Trends Parasitol. 2012;28:23-30.

26. Beeson JG, Drew DR, Boyle MJ, Feng G, Fowkes FJI, Richards JS. Merozoite surface proteins in red blood cell invasion, immunity and vaccines against malaria. FEMS Microbiol Rev. 2016;40:343-72.

27. Adams JH, Sim BK, Dolan SA, Fang X, Kaslow DC, Miller LH. A family of erythrocyte binding proteins of malaria parasites. Proc Natl Acad Sci USA. 1992;89:7085-9.

28. Adams JH, Blair PL, Kaneko O, Peterson DS. An expanding ebl family of Plasmodium falciparum. Trends Parasitol. 2001;17:297-9.

29. Orlandi PA, Klotz FW, Klotz FW, Haynes JD. A malaria invasion receptor, the 175-kilodalton erythrocyte binding antigen of Plasmodium falciparum recognizes the terminal Neu5Ac(alpha 2-3)Gal-sequences of glycophorin A. J Cell Biol. 1992;116:901-9.

30. Sim BK, Orlandi PA, Haynes JD, Klotz FW, Carter JM, Camus D, et al. Primary structure of the 175K Plasmodium falciparum erythrocyte binding antigen and identification of a peptide which elicits antibodies that inhibit malaria merozoite invasion. Cell Biol. 1990;1:1877-84.

31. Sim BKL, Chitnis CE, Wasniowska K, Hadley TJ, Miller LH. Receptor and ligand domains for invasion of erythrocytes by Plasmodium falciparum. Science. 1994;264:1941-4.

32. Sim BKL. EBA-175: An erythrocyte-binding ligand of Plasmodium falciparum. Parasitol Today. 1995;11:213-7.

33. Chowdhury P, Sen S, Kanjilal SD, Sengupta S. Genetic structure of two erythrocyte binding antigens of Plasmodium falciparum reveals a contrasting pattern of selection. Infect Genet Evol. 2018;57:64-74.

34. Daugherty JR, Murphy CI, Doros-Richert LA, Barbosa A, Kashala LO Ballou WR, et al. Baculovirus-mediated expression of Plasmodium falciparum erythrocyte binding antigen 175 polypeptides and their recognition by human antibodies. Infect Immun. 1997;65:3631-7.

35. Liang H, Narum DL, Fuhrmann SR, Luu T, Sim BK. A recombinant baculovirus-expressed Plasmodium falciparum receptor-binding domain of erythrocyte binding protein EBA-175 biologically mimics native protein. Infect Immun. 2000;68:3564-88.

36. Persson KE, Fowkes FJ, McCallum FJ, Gicheru N, Reiling L, Richards JS, et al. Erythrocyte-binding antigens of Plasmodium falciparum are targets of human inhibitory antibodies and function to evade naturally acquired immunity. J Immunol. 2013;191:785-94.

37. Bustamante LY, Powell GT, Lin YC, Macklin MD, Cross N, Kemp A, et al. Synergistic malaria vaccine combinations identified by systematic antigen screening. Proc Natl Acad Sci USA. 2017;114:12045-50.

38. Ochola-Oyier LI, Wamae K, Omedo I, Ogola C, Matharu A, Musayibimana JP, et al. Few Plasmodium falciparum merozoite ligand and erythrocyte receptor pairs show evidence of balancing selection. Infect Genet Evol. 2019;69:235-45.

39. Jakobsen $\mathrm{PH}$, Heegaard PM, Koch C, Wasniowska K, Lemnge MM, Jensen JB, et al. Identification of an erythrocyte binding peptide from the erythrocyte binding antigen, EBA-175, which blocks parasite multiplication and induces peptide-blocking antibodies. Infect Immun. 1998;66:4203-7

40. Okoyeh JN, Pillai CR, Chitnis CE. Plasmodium falciparum field isolates commonly use erythrocyte invasion pathways that are independent of sialic acid residues of glycophorin A. Infect Immun. 1999;67:5784-91.

41. Duraisingh MT, Maier AG, Triglia T, Cowman AF. Erythrocyte-binding antigen 175 mediates invasion in Plasmodium falciparum utilizing sialic acid-dependent and -independent pathways. Proc Natl Acad Sci USA. 2003;100:4796-801.

42. Tolia NH, Enemark EJ, Sim BKL, Joshua-Tor L. Structural basis for the EBA175 erytrocyte invasion pathway of the malaria parasite Plasmodium falciparum. Cell. 2005;122:183-93.
43. Bharara R, Singh S, Pattnaik P, Chitnis CE, Sharma A. Structural analogs of sialic acid interfere with the binding of erythrocyte binding antigen-175 to glycophorin A, an interaction crucial for erythrocyte invasion by Plasmodium falciparum. Mol Biochem Parasitol. 2004;138:123-9.

44. Salinas ND, Tolia NH. A quantitative assay for binding and inhibition of Plasmodium falciparum erythrocyte binding antigen 175 reveals high affinity binding depends on both DBL domains. Protein Expr Purif. 2014;95:188-94

45. Ambroggio $X$, Jiang L, Aebig J, Obiakor H, Lukszo J, Narum DL. The epitope of monoclonal antibodies blocking erythrocyte invasion by Plasmodium falciparum map to the dimerization and receptor glycan binding sites of EBA-175. PLOS ONE. 2013;8:e56326.

46. Wanaguru M, Crosnier C, Johnson S, Rayner JC, Wright GJ. Biochemical analysis of the Plasmodium falciparum erythrocyte-binding antigen-175 (EBA175)-glycophorin-A interaction: implications for vaccine design. J Biol Chem. 2013;288:32106-17.

47. Chen E, Paing MM, Salinas N, Sim BK, Tolia NH. Structural and functional basis for inhibition of erythrocyte invasion by antibodies that target Plasmodium falciparum EBA-175. PLoS Pathog. 2013;9:e1003390.

48. Salinas ND, Paing MM, Tolia NH. Critical glycosylated residues in exon three of erythrocyte glycophorin A engage Plasmodium falciparum EBA-175 and define receptor specificity. MBio. 2014;5:e01606-14.

49. Jaskiewicz E, Czerwinski M, Syper D, Lisowska E. Anti-M monoclonal antibodies cross-reacting with variant $\mathrm{Mg}$ antigen: an example of modulation of antigenic properties of peptide by its glycosylation. Blood. 1994;84:2340-54.

50. Cartron JP, Prou O, Luilier M, Soulier JP. Susceptibility to invasion by Plasmodium falciparum of some human erythrocytes carrying rare blood group antigens. Br J Haematol. 1983;55:639-47.

51. Bigham AW, Magnaye K, Dunn DM, Weiss RB, Bamshad M. Complex signatures of natural selection at GYPA. Hum Genet. 2018;137:151-60.

52. Baldwin MR, Li X, Hanada T, Liu SC, Chishti AH. Merozoite surface protein 1 recognition of host glycophorin A mediates malaria parasite invasion of red blood cells. Blood. 2015;125:2704-11.

53. Koch M, Wright KE, Otto O, Herbig M, Salinas ND, Tolia NH, et al. Plasmodium falciparum erythrocyte-binding antigen 175 triggers a biophysical change in the red blood cell that facilitates invasion. Proc Natl Acad Sci USA. 2017:114:4225-30.

54. Sisquella X, Nebl T, Thompson JK, Whitehead L, Malpede BM, Salinas ND. Plasmodium falciparum ligand binding to erythrocytes induce alterations in deformability essential for invasion. Elife. 2017;6:e21083.

55. Paing MM, Salinas ND, Adams Y, Oksman A, Jensen AT, Goldberg DE. Shed EBA-175 mediates red blood cell clustering that enhances malaria parasite growth and enables immune evasion. Elife. 2018;7:e43224.

56. Bigham AW, Magnaye K, Dunn DM, Weiss RB, Bamshad M. Complex signatures of natural selection at GYPA. Hum Genet. 2018;137:151-60.

57. Dolan SA, Miller LH, Wellems TE. Evidence for a switching mechanism in the invasion of erythrocytes by Plasmodium falciparum. J Clin Invest. 1990;86:618-24.

58. Gaur D, Storry JR, Reid ME, Barnwell JW, Miller LH. Plasmodium falciparum is able to invade erythrocytes through a trypsin-resistant pathway independent of glycophorin B. Infect Immun. 2003;71:6742-6.

59. Dolan SA, Proctor Jl, Alling DW, Okubo Y, Wellems TE, Miller LH. Glycophorin B as an EBA-175 independent Plasmodium falciparum receptor of human erythrocytes. Mol Biochem Parasitol. 1994;64:55-63.

60. Peterson DS, Wellems TE. EBL-1, a putative erythrocyte binding protein of Plasmodium falciparum, maps within a favored linkage group in two genetic crosses. Mol Biochem Parasitol. 2000;5:105-13.

61. Curtidor H, Rodríguez LE, Ocampo M, López R, García JE, Valbuena J, et al. Specific erythrocyte binding capacity and biological activity of Plasmodium falciparum erythrocyte binding ligand 1 (EBL-1)-derived peptides. Protein Sci. 2005;14:464-73.

62. Mayer DC, Cofie J, Jiang L, Hartl DL, Tracy E, Kabat J, et al. Glycophorin $B$ is the erythrocyte receptor of Plasmodium falciparum erythrocytebinding ligand, EBL-1. Proc Natl Acad Sci USA. 2009;106:5348-52.

63. Tarazona-Santos E, Castilho L, Amaral DR, Costa DC, Furlani NG, Zuccherato LW, et al. Population genetics of GYPB and association study between GYPB*S/s polymorphism and susceptibility to $P$ falciparum infection in the Brazilian Amazon. PLoS ONE. 2011;6:e16123. 
64. Fraser GR, Giblett ER, Motulsky AG. Population genetic studies in the Congo. 3. Blood groups (ABO, MNSs, Rh, Jsa). Am J Hum Genet. 1966;18:546-52

65. Li X, Marinkovic M, Russo C, McKnight CJ, Coetzer TL, Chishti AH. Identification of a specific region of Plasmodium falciparum EBL-1 that binds to host receptor glycophorin B and inhibits merozoite invasion in human red blood cells. Mol Biochem Parasitol. 2012;183:23-31.

66. Bianchin A, Bell A, Chubb AJ, Doolan N, Leneghan D, Stavropoulos I, et al. Design and evaluation of antimalarial peptides derived from prediction of short linear motifs in proteins related to erythrocyte invasion. PLoS ONE. 2015;10:e0127383.

67. Dankwa S, Chaand M, Kanjee U, Jiang RHY, Nobre LV, Goldberg JM, et al. Genetic evidence for erythrocyte receptor glycophorin B expression levels defining a dominant Plasmodium falciparum invasion pathway into human erythrocytes. Infect Immun. 2017;85:e00074.

68. Field SP, Hempelmann E, Mendelow BV, Fleming AF. Glycophorin variants and Plasmodium falciparum: protective effect of the Dantu phenotype in vitro. Hum Genet. 1994;93:148-50.

69. Tarazona-Santos E, Castilho L, Amaral DR, Costa DC, Furlani NG, Zuccherato LW, et al. Population genetics of GYPB and association study between GYPB*S/s polymorphism and susceptibility to $P$. falciparum infection in the Brazilian Amazon. PLoS ONE. 2011;6:e16123.

70. Malaria Genomic Epidemiology Network, Band G, Rockett KA, Spencer CC, Kwiatkowski DP. A novel locus of resistance to severe malaria in a region of ancient balancing selection. Nature. 2015;526:253-7.

71. Leffler EM, Band G, Busby GBJ, Kivinen K, Le QS, Clarke GM, et al. Resistance to malaria through structural variation of red blood cell invasion receptors. Science. 2017;356:eaam6393.

72. Rahuel C, Elouet JF, Cartron JP. Post-transcriptional regulation of the cell surface expression of glycophorins A, B, and E. J Biol Chem. 1994;269:32752-8.

73. Ndila CM, Uyoga S, Macharia AW, Nyutu G, Peshu N, Ojal J, et al. MalariaGEN Consortium Human candidate gene polymorphisms and risk of severe malaria in children in Kilifi, Kenya: a case-control association study. Lancet Haematol. 2018;5:e333-45.

74. Algady W, Louzada S, Carpenter D, Brajer P, Färnert A, Rooth I, et al. The malaria-protective human glycophorin structural variant DUP4 shows somatic mosaicism and association with hemoglobin levels. Am J Hum Genet. 2018;103:769-76.

75. Mayer DC, Kaneko O, Hudson-Taylor DE, Reid ME, Miller LH. Characterization of a Plasmodium falciparum erythrocyte-binding protein paralogous to EBA-175. Proc Natl Acad Sci USA. 2001;98:5222-7.

76. Thompson JK, Triglia T, Reed MB, Cowman AF. A novel ligand from Plasmodium falciparum that binds to a sialic acid-containing receptor on the surface of human erythrocytes. Mol Biol. 2001;41:47-58.

77. Narum DL, Fuhrmann SR, LuU T, Sim BK. A novel Plasmodium falciparum erythrocyte binding protein-2 (EBP2/BAEBL) involved in erythrocyte receptor binding. Mol Biochem. Parasitol. 2002;119:159-68.

78. Mayer DC, Mu JB, Feng X, Su XZ, Miller LH. Polymorphism in a Plasmodium falciparum erythrocyte-binding ligand changes its receptor specificity. J Exp Med. 2002;196:1523-52.

79. Jiang L, Duriseti S, Sun P, Miller LH. Molecular basis of binding of the Plasmodium falciparum receptor BAEBL to erythrocyte receptor glycophorin C. Mol Biochem Parasitol. 2009;168:49-54.

80. Maier AG, Baum J, Smith B, Conway DJ, Cowman AF. Polymorphisms in erythrocyte binding antigens 140 and 181 affect function and binding but not receptor specificity in Plasmodium falciparum. Infect Immun. 2009;77:1689-99.

81. Maier AG, Duraisingh MT, Reeder JC, Patel SS, Kazura JW, Zimmerman PA, et al. Plasmodium falciparum erythrocyte invasion through glycophorin $C$ and selection for Gerbich negativity in human populations. Nat Med. 2003;9:87-92.

82. Rydzak J, Kaczmarek R, Czerwinski M, Lukasiewicz J, Tyborowska J, Szewczyk $B$, et al. The baculovirus-expressed binding region of Plasmodium falciparum EBA-140 ligand and its glycophorin C binding specificity. PLOS ONE. 2015;10:e0115437.
83. Lobo CA, Rodriguez M, Reid M, Lustigman S. Glycophorin C is the receptor for the Plasmodium falciparum erythrocyte binding ligand PfEBP-2 (baebl). Blood. 2003;101:4628-31.

84. Zimmerman PA, Patel SS, Maier AG, Bockarie MJ, Kazura JW. Erythrocyte polymorphisms and malaria parasite invasion in Papua New Guinea. Trends Parasitol. 2003;19:250-2.

85. Mayer DC, Jiang L, Achur RN, Kakizaki I, Gowda DC, Miller LH. The glycophorin C N-linked glycan is a critical component of the ligand for the Plasmodium falciparum erythrocyte receptor BAEBL. Proc Natl Acad Sci USA. 2006;103:2358-62.

86. Lin DH, Malpede BM, Batchelor JD, Tolia NH. Crystal and solution structures of Plasmodium falciparum erythrocyte-binding antigen 140 reveal determinants of receptor specificity during erythrocyte invasion. J Biol Chem. 2012;287:36830-6.

87. Malpede BM, Lin DH, Tolia NH. Molecular basis for sialic aciddependent receptor recognition by the Plasmodium falciparum invasion protein erythrocyte-binding antigen-140/BAEBL. J Biol Chem. 2013;288:12406-15.

88. Batchelor JD, Zahm JA, Tolia NH. Dimerization of Plasmodium vivax DBP is induced upon receptor binding and drives recognition of DARC. Nat Struct Mol Biol. 2011;18:908-14.

89. Batchelor JD, Malpede BM, Omattage NS, DeKoster GT, HenzlerWildman KA, Tolia NH. Red blood cell invasion by Plasmodium vivax: structural basis for DBP engagement of DARC. PLoS Pathog. 2014;10:e1003869.

90. Ashline DJ, Duk M, Lukasiewicz J, Reinhold VN, Lisowska E, Jaskiewicz $E$. The structures of glycophorin $C N$-glycans, a putative component of the GPC receptor site for Plasmodium falciparum EBA-140 ligand. Glycobiology. 2015;25:570-81.

91. Yiangou L, Montandon R, Modrzynska K, Rosen B, Bushell W, Hale C, et al. A stem cell strategy identifies glycophorin $C$ as a major erythrocyte receptor for the rodent malaria parasite Plasmodium berghei. PLoS ONE. 2016;11:e0158238.

92. Wahlgren M, Goel S, Akhouri RR. Variant surface antigens of Plasmodium falciparum and their roles in severe malaria. Nat Rev Microbiol. 2017;15:479-91.

93. Sherman IW, Eda S, Winograd E. Cytoadherence and sequestration in Plasmodium falciparum: defining the ties that bind. Microbes Infect. 2003;5:897-909.

94. Janes JH, Wang CP, Levin-Edens E, Vigan-Womas I, Guillotte M, Melcher $M$, et al. Investigating the host binding signature on the Plasmodium falciparum PfEMP1 protein family. PLOS Pathog. 2011;7:1002032.

95. Niang M, Bei AK, Madnani KG, Pelly S, Dankwa S, Kanjee U, et al. STEVOR is a Plasmodium falciparum erythrocyte binding protein that mediates merozoite invasion and rosetting. Cell Host Microbe. 2014;16:81-93.

96. Lee W-C, Malleret B, Lau Y-L, Mauduit M, Fong M-Y, Cho JS, et al. Glycophorin C (CD236R) mediates vivax malaria parasite rosetting to normocytes. Blood. 2014;123:e100-9.

97. Zerka A, Rydzak J, Lass A, Szostakowska B, Nahorski W, Wroczyńska A, et al. Studies on immunogenicity and antigenicity of baculovirusexpressed binding region of Plasmodium falciparum EBA-140 merozoite ligand. Arch Immunol Ther Exp (Warsz). 2016;64:149-56.

98. Satchwell TJ. Erythrocyte invasion receptors for Plasmodium falciparum: new and old. Transfus Med. 2016;26:77-88.

99. Zerka A, Olechwier A, Rydzak J, Kaczmarek R, Jaskiewicz E. Baculovirusexpressed Plasmodium reichenowi EBA-140 merozoite ligand is host specific. Parasitol Int. 2016;65:708-14.

100. Zerka A, Kaczmarek R, Czerwinski M, Jaskiewicz E. Plasmodium reichenowi EBA-140 merozoite ligand binds to glycophorin D on chimpanzee red blood cells, shedding new light on origins of Plasmodium falciparum. Parasit Vectors. 2017;10:554.

\section{Publisher's Note}

Springer Nature remains neutral with regard to jurisdictional claims in published maps and institutional affiliations. 